\title{
Role of image-defined risk factors in predicting surgical complications of localized neuroblastoma
}

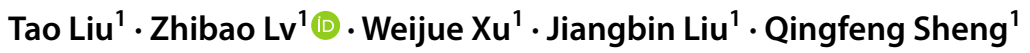

Accepted: 12 August 2020 / Published online: 26 August 2020

c) Springer-Verlag GmbH Germany, part of Springer Nature 2020

\begin{abstract}
Purpose To explore the relationship between image-defined risk factors and surgical complications of localized neuroblastoma.

Methods We retrospectively evaluated 84 patients who met the inclusion criteria at our hospitals between June 2014 and June 2019. Patients' clinic data were collected and the common terminology criteria for adverse events were used to categorize complications as major (grade 3-4) or minor (grade 1-2).

Results Four (11.8\%) out of 34 stage L1 patients and 15 (30.0\%) out of 50 stage L2 patients had surgical complications $(P<0.05)$. Seventy patents underwent primary surgery, including all stage $\mathrm{L} 1$ patients and 36 stage $\mathrm{L} 2$ patients. There were no significant differences between the two groups regarding surgical complications or major surgical complications. Among stage L2 patients, $2(5.6 \%)$ out of 36 who underwent primary surgery and $2(14.3 \%)$ out of 14 who underwent secondary surgery had major surgical complications $(P<0.05)$. Complete tumor resection was achieved in $18(50 \%)$ and $7(50 \%)$ patients in each group $(P>0.05)$. The mean numbers of IDRFs were 2.06 and 4.29 , respectively $(P<0.05)$.

Conclusions Localized neuroblastoma patients with IDRFs have a greater surgical risk. And the number of IDRFs is not ignorable, especially in predicting major surgical complications.
\end{abstract}

Keywords Neuroblastomas $\cdot$ IDRFS $\cdot$ Neoadjuvant chemotherapy $\cdot$ Surgical complication

\section{Introduction}

Neuroblastic tumors (neuroblastomas, ganglioneuroblastomas, and ganglioneuromas) are the commonest extracranial solid tumors that afflict children [1]. To develop a consensus approach to pretreatment risk stratification, a new international neuroblastoma risk group (INRG) staging system (INRGSS) was designed to stratify patients prior to implementation of any treatment (including surgery) [1, 2]. Locoregional tumors are staged $\mathrm{L} 1$ or $\mathrm{L} 2$ based on the absence or presence of one or more of 20 image-defined risk factors (IDRFs), respectively. Stage M tumors refer to distant metastatic disease (not contiguous with the primary tumor), except when defined as stage MS. Stage MS tumors refer to metastatic disease in patients younger than 18 months

Zhibao Lv

zhibaolyu@163.com

1 Department of General Surgery, Children's Hospital of Shanghai, Shanghai Jiao Tong University, Shanghai 200040, China
(547 days) with metastasis confined to the skin, liver, and/ or bone marrow ( $<10 \%$ tumors) [2].

In 2005, the first Localized Neuroblastoma European Study conducted by the European International Society of Pediatric Oncology Neuroblastoma Group offered specific surgical guidelines for the management of localized neuroblastoma. Surgical risk factors (which were adapted by the INRG as IDRFs) were defined to avoid operations likely to result in complications or gross residual disease. An attempt at resection was recommended only if these factors were excluded by preoperative imaging [3]. Later, other publications also reported that IDRFs can be used as an aid to identify patients who are suitable for surgery as the first treatment, hereafter termed primary surgery. The absence of IDRFs (stage L1) would predict a low risk of surgical complications and a high possibility of complete tumor excision. In the presence of IDRFs (stage L2), the guideline advised against primary surgery. In this situation, surgery was to be limited to biopsy with the expectation of a more safe and effective excision after neoadjuvant chemotherapy [4-6]. However, Yoneda et al. reported that the surgical 
complication rate remained the same regardless of whether neoadjuvant chemotherapy reduced the number of IDRFs or not [7]. Simon et al. found that the presence of IDRFs did not indicate unresectability and could not improve the predicted outcome of localized neuroblastoma [6]. Fumino et al. also proposed that IDRFs may overestimate the surgical risks, leading to unnecessary chemotherapy and prolonged stay in hospital [8].

Thus, the role of image-defined risk factors in predicting surgical complications of localized neuroblastoma is still controversial.

\section{Materials and methods}

Ethical approval was obtained from the Ethics Boards of Shanghai Children's Hospital. We retrospectively evaluated 171 patients diagnosed with neuroblastomas at our hospitals in China between June 2014 and June 2019. Among them, 84 patients with localized neuroblastoma met our inclusion criteria: image data were available at diagnosis and before surgery; radical surgery was performed at our hospital to obtain information about the surgery. The flow chart of patient selection in the present study is shown in Fig. 1.

One pediatric surgeon and one pediatric radiologist at our hospital evaluated all images directly. We assessed IDRFs both at diagnosis and preoperatively on contrast-enhanced computed tomography scan and added magnetic resonance imaging information if necessary. The IDRFs were defined according to the new guideline published in 2011 [2]. According to the treatment regimen for neuroblastoma in our hospital, localized patients are divided into low-risk, middle-risk and high-risk group based on age, MYCN status, and INSS stage. Middle-risk group and high-risk group patients received chemotherapy before or after surgery. And the surgeon decided when to surgery according to imaging characteristics of tumors. The operations were performed by the chief surgeons of the Departments of General Surgery, Cardiothoracic Surgery, or Neck Surgery depending on the primary site of the tumor. Common terminology criteria for adverse events were used to categorize complications as major (grade 3-4) or minor (grade 1-2) [9]. Complete surgical resection was defined as no visible/palpable disease which was determined by the individual operating surgeon and noted in the surgical checklist.

Descriptive statistics were reported as absolute frequencies and percentages for qualitative variables or medians and ranges for continuous variables. All statistical analyses were performed using Stata version 20.0 (StataCorp LP, College Station, TX, USA). Analysis items with a $P$ value of $<0.05$ were considered statistically significant.

\section{Results}

Eighty-four patients were enrolled in the present study. The background characteristics of these patients are shown in Table 1. The median age was 40 months (range 1-226) and more than $75 \%$ of them were younger than 60 months. Thirty-four and 50 patients were diagnosed with stage L1 and stage L2 disease, respectively. Among the patients with stage L2 disease, 36 underwent primary surgery and 14 received neoadjuvant chemotherapy. The most often occurring complication was chyloabdomen or chylothorax in six patients (four in stage L2 patients who got primary surgery, two in stage L2 patients who got secondary surgery), then Horner syndrome in five patients (three in stage L2 patients who got primary surgery, two in stage L1 patients), pneumothorax in four patients ( 2 in stage L1 patients, 2 in stage L2 patients who got primary surgery), injury of the renal blood

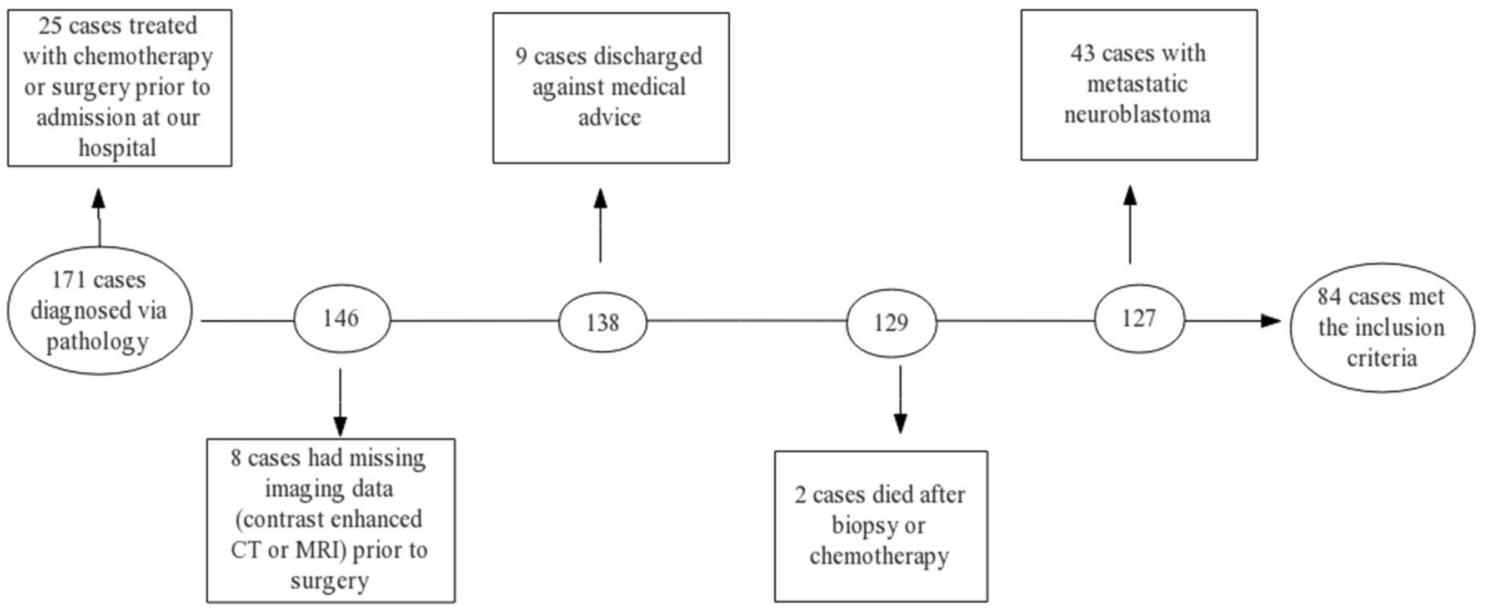

Fig. 1 Flowchart of patient selection in the present study 
Table 1 Distribution of patients' characteristics

\begin{tabular}{llll}
\hline Items & Results & Items & Results \\
\hline Gender (cases) & $\mathrm{M}(n=40) ; \mathrm{F}(n=44)$ & Complications, $n$ & Major/minor \\
Age (months), median (range) & $40(1-226) ; 75 \%<60$ & Chyloabdomen or chylothorax & $0 / 6$ \\
Site, $n(\%)$ & & Horner syndrome & $0 / 5$ \\
Neck & $4(4.8)$ & Pneumothorax & $0 / 4$ \\
Cervicothoracic junction & $2(2.4)$ & Injury of the renal blood vessels & $2 / 0$ \\
Thorax & $30(35.7)$ & Injury of the inferior vena cava & $1 / 0$ \\
Abdomen and pelvis & $48(57.1)$ & Paralysis & $1 / 0$ \\
INRG stage, $n(\%)$ & & Pathology, $n(\%)$ & \\
L1 & $34(40.5)$ & Neuroblastoma & $44(52.4)$ \\
L2 & $50(59.5)$ & Ganglioneuroblastoma & $32(38.1)$ \\
MYCN status & & Ganglioneuroma & $8(9.5)$ \\
Amplification & $34(40.5)$ & & \\
No amplification & $4(0.5)$ & & \\
\hline
\end{tabular}

vessels in two patients (one in stage L2 patients who got primary surgery, another one in stage L2 patients who got secondary surgery), injury of the inferior vena cava in one stage L2 patients who got secondary surgery, and paralysis in one stage L2 patient who got primary surgery.

We categorized the patients into different groups (Table 2). Out of 84 patients with local neuroblastoma, 19 (22.6\%) patients had surgical complications. Patients with stage L2 disease were likelier to have surgical complications than those with stage L1 disease, and the difference was statistically significant. However, there were no significant differences in the distribution of major surgical complications. Among them, 70 patents underwent primary surgery, including all patients with stage L1 disease and 36 patients with stage L2 disease. There were no significant differences between the two groups regarding surgical complications or major surgical complications.
Among 50 patients with stage L2 disease, 36 underwent primary surgery and $5.6 \%$ of them had major surgical complications. The remaining patients underwent secondary surgery and $14.3 \%$ of them had major surgical complications. Between these two groups, there was a significant difference in the distribution of major surgical complications but not surgical complications.

Subsequently, we divided patients with stage L2 disease into two groups (Table 3 ). One group underwent primary surgery while the other underwent secondary surgery. Complete tumor resection was achieved in $50 \%$ of patients in each group, with no statistically significant difference. The mean numbers of IDRFs were 2.06 for patients who underwent primary surgery, and the mean numbers of IDRFs were 4.29 before neoadjuvant chemotherapy for patients who underwent secondary surgery, with a significant difference $(P<0.05)$

Table 2 Distribution of complications of radical surgery (surgery for treatment, not diagnosis)

\begin{tabular}{|c|c|c|c|c|c|c|c|c|c|}
\hline & & No complications ${ }^{\mathrm{a}}$ & Complications & Total & $P$ value & $\begin{array}{l}\text { No major } \\
\text { complica- } \\
\text { tion }\end{array}$ & Major complication & Total & $P$ value \\
\hline \multirow[t]{3}{*}{ INRG, $n(\%)$} & $\mathrm{L} 1$ & $30(88.2)$ & $4(11.8)$ & $34(100)$ & 0.043 & $34(100)$ & $0(0.0)$ & $34(100)$ & $>0.05$ \\
\hline & L2 & $35(70.0)$ & $15(30.0)$ & $50(100)$ & & $46(92.0)$ & $4(8.0)$ & $50(100)$ & \\
\hline & Total & $65(77.4)$ & $19(22.6)$ & $84(100)$ & & $80(95.2)$ & $4(4.8)$ & $84(100)$ & \\
\hline \multirow[t]{3}{*}{$\mathrm{INRG}^{\mathrm{b}}, n(\%)$} & L1 & $30(88.2)$ & $4(11.8)$ & $34(100)$ & $>0.05$ & $34(100)$ & $0(0.0)$ & $34(100)$ & $>0.05$ \\
\hline & L2 & $25(69.5)$ & $11(30.5)$ & $36(100)$ & & $34(94.4)$ & $2(5.6)$ & $36(100)$ & \\
\hline & Total & 55 (78.6) & $15(21.4)$ & $70(100)$ & & $68(97.1)$ & $2(2.9)$ & $70(100)$ & \\
\hline \multicolumn{10}{|l|}{ INRG L2, $n(\%)$} \\
\hline Primary surgery & & $25(69.4)$ & $11(30.6)$ & $36(100)$ & $>0.05$ & $34(94.4)$ & $2(5.6)$ & $36(100)$ & 0.031 \\
\hline Secondary surgery & & $10(71.4)$ & $4(28.6)$ & $14(100)$ & & $12(85.7)$ & $2(14.3)$ & $14(100)$ & \\
\hline Total & & $35(70.0)$ & $15(30.0)$ & $50(100)$ & & $46(92.0)$ & $4(8.0)$ & $50(100)$ & \\
\hline
\end{tabular}

${ }^{\mathrm{a}}$ Complications referred to major and minor surgical complications

${ }^{\mathrm{b}}$ INRG L2 patients who underwent primary surgery 
Table 3 Distribution of extent of surgery and the number of IDRFs among patients with stage L2 neuroblastoma

\begin{tabular}{lllll}
\hline & Primary surgery & Secondary surgery & Total & $P$ value \\
\hline Complete resection, $n(\%)$ & $7(50)$ & $18(50)$ & $25(100)$ & $>0.05$ \\
Incomplete resection, $n(\%)$ & $7(50)$ & $18(50)$ & $25(100)$ & \\
Total, $n(\%)$ & $14(100)$ & $36(100)$ & $50(100)$ & \\
Mean number of IDRFs & 2.06 & 4.29 & & 0.000 \\
\hline
\end{tabular}

\section{Discussion}

In our study, the surgical complication rates were $11.8 \%$ and 30\% among patients with stages L1 and L2 disease, respectively. These results are similar to the respective rates of $15.5 \%$ and $33.3 \%$ reported by Yoneda et al. [10]. Another study that emanated from Germany reported respective rates of $14.5 \%$ and $26.6 \%$ [6]. We excluded 14 patients with stage L2 disease who received neoadjuvant chemotherapy, and found surgical complication rates of $30.5 \%$ and $11.8 \%$ among the remaining patients with stages $\mathrm{L} 2$ and $\mathrm{L} 1$ disease, respectively $(P>0.05)$. This suggests that the presence or absence of IDRFs may not indicate foolproof safe surgery.

To date, most pieces of evidence suggest that stage L1 tumors could be safely resected and that for others, a decrease in IDRFs along with shrinkage after chemotherapy can predict better rates of resection and lower complication rates $[3-5,11]$. Irtan et al. reported that the degree of decrease was greater for patients with stages M and MS disease (61.5\%) than for patients with stage L2 disease (38.5\%) [12]. However, we found that there were no significant differences regarding surgical complications or major surgical complications between stage L1 patients and stage L2 patients who got primary surgery. Besides, secondary surgery was more prone to major surgical complications for stage L2 patients, which indicates that not only the presence, but the number or type of IDRFs may matter. Consistent with our hypothesis, the mean numbers of IDRFs were 2.06 and 4.29 before neoadjuvant chemotherapy, respectively, among patients who underwent primary and secondary surgery, with a significant difference. There was no significant difference in the correlation of each IDRF and the occurrence of surgical complications. However, Zhang et al. found that not all IDRFs are of equal importance. It was less likely to perform complete resection of the tumor with respect to the following IDRFs: compressed trachea, encased aorta, intraspinal tumor extension, encased celiac axis, and encased superior mesenteric artery [13]. However, the authors did not assess the surgical complications. We found that there was no significant difference in the extent of tumor resection between the two groups. A significantly negative correlation between the number of IDRFs after neoadjuvant chemotherapy and the possibility of complete tumor resection was mentioned in other reports [13, 14]. The difference may be due to the different extents of tumor resection [13-15].
Avanzini et al. analyzed the results of the SIOPEN study among patients with stage L2 disease and found complete resection was more successful among children who had disappearance or numerical decreases in IDRFs [16].

The INRG staging system was established by the INRG task force in 2009 [1, 17]. In 2011, a new guideline for assessing IDRFs was published [2]. According to the new guideline, the situation in which "the tumor is only in contact with renal vessels" should be considered as "IDRF present"; previously, this was diagnosed as "IDRF absent". According to the study conducted by Yoneda et al., the new guideline increased the ratio of IDRF-present patients from 31 to $71 \%$ and improved its sensitivity but reduced the specificity and accuracy. Although the presence of IDRFs after the new guideline was significantly associated with surgical complications, a considerable number of patients with stage L2 disease had undergone chemotherapy after application of the new guideline, who could have safely been cured by surgery alone. The authors also found that preoperative chemotherapy did not decrease the frequency of surgical complications among patients [10].

The SIOPEN infant neuroblastoma study reported that 35-40\% patients could experience a decrease in IDRFs after neoadjuvant chemotherapy. Another study reported a rate of decrease of $60 \%$. Only $27 \%$ of patients with stage L2 tumors had no IDRFs after preoperative chemotherapy [7, 17]. Thus, patients with stage L2 tumors may have a potential risk for surgery even after neoadjuvant chemotherapy [18]. Also, some aspects of the operation only become obvious during the procedure, despite meticulous preoperative evaluation [19]. Further, Monclair et al. conducted a study of 243 patients with stage L2 disease without MYCN amplification. The authors found 5-year event-free survival (EFS) and 5 -year overall survival (OS) rates of $86 \%$ and $95 \%$, respectively, among 118 patients who underwent primary surgery, which were significantly better than the rates found among patients who did not undergo primary surgery $(73 \%$ vs $83 \%$, respectively) $(P<0.0025), 106$ of whom received neoadjuvant chemotherapy. The authors doubted that patients with stage L2 disease who underwent primary surgery would have fared better with neoadjuvant chemotherapy. They also found that patients who underwent primary surgery had significantly fewer IDRFs and significantly lower International Neuroblastoma stage 3 disease than patients with stage L2 disease who underwent neoadjuvant chemotherapy [20]. 
A limitation of our study was that it was a retrospective study conducted at a single institution in a developing country with a relatively small study population. A multicenter and prospective trial with a larger study population is needed to verify the value of IDRFs.

\section{Conclusions}

Surgery remains the mainstay of treatment for pediatric neuroblastomas; however, every effort should be made to plan a safe and effective operation. Localized neuroblastoma patients with IDRFs have a greater surgical risk. And the number of IDRFs is not ignorable, especially in predicting major surgical complications. For some part of stage L2 patients, the number of the IDRFs may play a more important role in the treatment regime.

Acknowledgements We thank Mr. Rui Liu and our colleagues for their help during the COVID-19 pandemic.

Author contributions TL and QS had the idea for the article, TL performed the data acquisition, data analysis, data interpretation, and manuscript preparation. ZLv, WX, and JL contributed to the critical review of the intellectual content of this manuscript.

Funding There is no funding.

\section{Compliance with ethical standards}

Conflict of interest The authors declare that they have no conflict of interest.

Ethical approval This retrospective chart review study involving human participants was in accordance with the ethical standards of the institutional and national research committee and with the 1964 Helsinki Declaration and its later amendments or comparable ethical standards. The Ethics Boards of Shanghai Children's Hospital approved this study.

\section{References}

1. Cohn SL, Pearson AD, London WB, Monclair T, Ambros PF, Brodeur GM et al (2009) The international neuroblastoma risk group (INRG) classification system: an INRG task force report. J Clin Oncol 27:289-297. https://doi.org/10.1200/jco.2008.16.6785

2. Brisse HJ, McCarville MB, Granata C, Krug KB, Wootton-Gorges SL, Kanegawa $\mathrm{K}$ et al (2011) Guidelines for imaging and staging of neuroblastic tumors: consensus report from the international neuroblastoma risk group project. Radiology 261:243-257. https ://doi.org/10.1148/radiol.11101352

3. Cecchetto G, Mosseri V, De Bernardi B, Helardot P, Monclair $\mathrm{T}$, Costa E et al (2005) Surgical risk factors in primary surgery for localized neuroblastoma: the LNESG1 study of the European international society of pediatric oncology neuroblastoma group. J Clin Oncol 23:8483-8489. https://doi.org/10.1200/ jco.2005.02.4661

4. Kushner BH, LaQuaglia MP, Kramer K, Cheung NV (2004) Radically different treatment recommendations for newly diagnosed neuroblastoma: pitfalls in assessment of risk. J Pediatr Hematol Oncol 26:35-39. https://doi.org/10.1097/00043426200401000-00012

5. Rubie H, De Bernardi B, Gerrard M, Canete A, Ladenstein R, Couturier J et al (2011) Excellent outcome with reduced treatment in infants with nonmetastatic and unresectable neuroblastoma without MYCN amplification: results of the prospective INES 99.1. J Clin Oncol 29:449-455. https://doi.org/10.1200/ jco.2010.29.5196

6. Simon T, Hero B, Benz-Bohm G, von Schweinitz D, Berthold F (2008) (2008) Review of image defined risk factors in localized neuroblastoma patients: results of the GPOH NB97 trial. Pediatr Blood Cancer 50:965-969. https://doi.org/10.1002/pbc.21343

7. Yoneda A, Nishikawa M, Uehara S, Oue T, Usui N, Inoue M et al (2016) Can neoadjuvant chemotherapy reduce the surgical risks for localized neuroblastoma patients with image-defined risk factors at the time of diagnosis? Pediatr Surg Int 32:209214. https://doi.org/10.1007/s00383-016-3858-5

8. Fumino S, Kimura K, Iehara T, Nishimura M, Nakamura S, Souzaki R et al (2015) Validity of image-defined risk factors in localized neuroblastoma: a report from two centers in Western Japan. J Pediatr Surg 50:2102-2106. https://doi.org/10.1016/j. jpedsurg.2015.08.039

9. Common terminology criteria for adverse events (CTCAE) V5.0. (2017)

10. Yoneda A, Nishikawa M, Uehara S, Oue T, Usui N, Inoue M et al (2016) Can image-defined risk factors predict surgical complications in localized neuroblastoma? Eur J Pediatr Surg 26:117-122. https://doi.org/10.1055/s-0035-1566100

11. Lezama-Del VP, Krauel L, LaQuaglia MP (2019) Error traps and culture of safety in pediatric surgical oncology. Semin Pediatr Surg 28:164-171. https://doi.org/10.1053/j.sempedsurg .2019.04.014

12. Irtan S, Brisse HJ, Minard-Colin V, Schleiermacher G, Galmiche-Rolland L, Le Cossec C et al (2015) Image-defined risk factor assessment of neurogenic tumors after neoadjuvant chemotherapy is useful for predicting intra-operative risk factors and the completeness of resection. Pediatr Blood Cancer 62:1543-1549. https://doi.org/10.1002/pbc.25511

13. Zhang A, Pan C, Xu M, Wang X, Ye Q, Gao Y et al (2019) (2019) Association of image-defined risk factors, tumor resectability, and prognosis in children with localized neuroblastoma. World J Pediatr 15:572-579. https://doi.org/10.1007/s1251 9-019-00274-y

14. Gunther P, Holland-Cunz S, Schupp CJ, Stockklausner C, Hinz U, Schenk JP (2011) Significance of image-defined risk factors for surgical complications in patients with abdominal neuroblastoma. Eur J Pediatr Surg 21:314-317. https://doi. org/10.1055/s-0031-1280824

15. Kubota M (2010) The role of surgery in the treatment of neuroblastoma. Surg Today 40:526-532. https://doi.org/10.1007/s0059 5-009-4092-8

16. Avanzini S, Pio L, Erminio G, Granata C, Holmes K, Gambart $M$ et al (2017) Image-defined risk factors in unresectable neuroblastoma: SIOPEN study on incidence, chemotherapy-induced variation, and impact on surgical outcomes. Pediatr Blood Cancer 64:e26605. https://doi.org/10.1002/pbc.26605

17. Monclair T, Brodeur GM, Ambros PF, Brisse HJ, Cecchetto G, Holmes K et al (2009) The international neuroblastoma risk group (INRG) staging system: an INRG task force report. J Clin Oncol 27:298-303. https://doi.org/10.1200/jco.2008.16.6876

18. Rich BS, McEvoy MP, Kelly NE, Oh E, Abramson SJ, Price AP et al (2011) Resectability and operative morbidity after chemotherapy in neuroblastoma patients with encasement of major visceral arteries. J Pediatr Surg 46:103-107. https://doi. org/10.1016/j.jpedsurg.2010.09.075 
19. Kohler JA, Rubie H, Castel V, Beiske K, Holmes K, Gambini C et al (2013) Treatment of children over the age of one year with unresectable localised neuroblastoma without MYCN amplification: results of the SIOPEN study. Eur J Cancer 49:3671-3679. https://doi.org/10.1016/j.ejca.2013.07.002

20. Monclair T, Mosseri V, Cecchetto G, De Bernardi B, Michon J, Holmes K (2015) Influence of image-defined risk factors on the outcome of patients with localised neuroblastoma. A report from the LNESG1 study of the European international society of paediatric oncology neuroblastoma group. Pediatr Blood Cancer 62:1536-1542. https://doi.org/10.1002/pbc.25460

Publisher's Note Springer Nature remains neutral with regard to jurisdictional claims in published maps and institutional affiliations. 